\title{
A protocol for managing dissociative symptoms in refugee populations
}

\author{
Zoe J. Chessell ${ }^{1}$, Francesca Brady ${ }^{1,2 *}$, Sameena Akbar ${ }^{1}$, Adele Stevens ${ }^{1,3}$ and Kerry Young ${ }^{1,4}$ \\ ${ }^{1}$ Woodfield Trauma Service, 7a Woodfield Road, London W9 2NW, UK, ${ }^{2}$ Helen Bamber Foundation, Baynes Street, London \\ NW1 0TF, UK, ${ }^{3}$ Traumatic Stress Service, 25 Erleigh Road, Reading, Berkshire RG1 5LR, UK and ${ }^{4}$ Oxford Rose Clinic, \\ John Radcliffe Hospital, Headley Way, Headington, Oxford OX3 9DU, UK \\ *Corresponding author. Email: Francesca@helenbamber.org
}

(Received 19 May 2018; revised 18 February 2019; accepted 20 February 2019)

\begin{abstract}
This article describes a clinical protocol for supporting those presenting with post-traumatic stress disorder (PTSD) and dissociative symptoms, particularly dissociative flashbacks, based on a cross-culturally applicable model. The protocol is discussed from the perspective of working with a refugee and asylum seeker population, although many of the principles will be applicable to clients from any background presenting with these dissociative symptoms. The protocol addresses the assessment and formulation of a client's dissociative symptoms. It includes guidance on sharing psycho-education with clients regarding the evolutionary function of dissociation and developing practical strategies to monitor and manage dissociative symptoms. The strengths and limitations of this protocol are also discussed.
\end{abstract}

\section{Key learning aims}

After reading this article people will:

(1) Be able to understand a cross-culturally applicable model of dissociation and how it applies to clinical practice when working with clients presenting with dissociative symptoms, particularly dissociative flashbacks, in the context of a diagnosis of PTSD.

(2) Be able to assess and formulate dissociative symptoms as part of an overall PTSD formulation.

(3) Be able to develop practical strategies for assisting clients in monitoring and managing their dissociative symptoms.

(4) Be familiar with adaptations for using this approach with refugee and asylum seeker populations.

Keywords: BAME; cross-cultural; dissociation; post-traumatic stress disorder; trauma

\section{Introduction}

The Diagnostic and Statistical Manual of Mental Disorders, Fourth Edition (DSM-IV) [American Psychiatric Association (APA), 1994; p. 477] defines dissociation as a 'disruption in the usually integrated functions of consciousness, memory, identity or perception of the environment'. It may manifest as a subjective feeling of altered perceptions in terms of time, place and self (Schauer and Elbert, 2010). Due to dissociation, memory encoding of a traumatic event can be disrupted (Brewin, 2001; Ehlers and Clark, 2000) and recall of the traumatic events may also trigger the associated peri-traumatic dissociative response (Schauer and Elbert, 2010). Peri-traumatic dissociation has been identified as a strong predictive factor for the development of posttraumatic stress disorder (PTSD) after a trauma (Birmes et al., 2003; Breh and Seidler, 2007; Ozer et al., 2003) and persistent post-traumatic dissociation has also been shown to play a role in the development of PTSD (Murray et al., 2002). 
Dissociation is particularly common in certain types of trauma, particularly more 'severe' traumatic experiences (Kennerley, 2009). The DSM-5 (APA, 2013) links dissociation to an overwhelming threat to a perceived inescapable event, such as childhood sexual abuse, torture, or war trauma (Schauer and Elbert, 2010). Schauer and Elbert (2010) suggest that dissociation is more likely to occur during traumatic events in which there is no chance of escape, particularly if the person has a history of multiple traumas. Literature suggests that children who have experienced abuse, particularly sexual abuse, are highly likely to dissociate at the time of the trauma, and later when reminded of the event, as a way of coping with the experience (Ainscough and Toon, 2000; Chu, 1998; Herman, 1992). Zatzick et al. (1994) found that veterans with higher exposure to war zone stress experienced higher levels of dissociation. Carlson and Rosser-Hogan (1991) found a relationship between the amount of trauma experienced and higher levels of dissociation in Cambodian refugees.

Many refugees have experienced prolonged or multiple traumatic events and may also have a history of trauma in childhood. This type of trauma history is likely to lead to more complex mental health problems, including high levels of dissociation. For ease of reading, the term 'refugee' is used throughout this article to describe vulnerable migrants, including asylum seekers or survivors of trafficking who may be in the process of claiming asylum, those who have been granted Refugee Status, and those who have a background of other types of forced migration.

Research shows that refugees present with higher levels of PTSD and depression than those from their host countries, although prevalence estimates vary (Bogic et al., 2015; Tinghög, 2017). While there is debate around the cross-cultural validity of the PTSD diagnosis (Patel et al., 2016; Summerfield, 2001), there is also evidence that it is cross-culturally valid (Hinton and Lewis-Fernández, 2011) and the evidence base for effective treatments for PTSD in refugee groups is ever growing (Nosè et al., 2017; Thompson et al., 2018). Furthermore, most refugees will have experienced significant post-migration difficulties such as destitution, homelessness or unstable accommodation, immigration uncertainty, bereavement and other losses, racism, and social isolation (Bhugra and Becker, 2005; Bogic et al., 2012; Carswell et al., 2009; Li et al., 2016; Porter and Haslam, 2005). These post-migration difficulties may exacerbate existing mental health problems or may contribute to the development of additional psychological difficulties (Bogic et al., 2012; Miller and Rasmussen, 2010). Due to these factors, research and clinical experience suggest that the likelihood of refugees experiencing dissociation is high (Carlson and Rosser-Hogan, 1991, 1994).

\section{A cross-cultural approach to dissociation}

A number of different models and theories exist to explain dissociation and its manifestations (which is beyond the scope of this article) and it continues to be ill-defined (Kennedy et al., 2013; Kennerley, 2009; Lewis-Fernandez et al., 2007). Schauer and Elbert (2010) suggest one possible explanatory model, the ' 6 Fs' cascade (see Fig. 1), which explains dissociation as an adaptive process that has its foundations in an evolutionary perspective of physiological responses to trauma. This model builds on Lang et al.'s (2000) proposed defence cascade, which consists of the psychological and physiological 'uproar' responses to threat of: 'Freeze', 'Flight' and 'Fight'. Schauer and Elbert (2010) propose extending the defence cascade to explain what occurs when a person is unable to escape a threat and suggests that it is evolutionarily adaptive to dissociate when escape from a threat is not possible. They propose three further 'shut-down' phases of response to threat that occur when an individual is unable to escape of: 'Fright', 'Flag' and 'Faint'.

Schauer and Elbert (2010) argue that dissociative 'shut-down' promotes preservation of life in the face of extreme threat. For example, if the individual has sustained a physical injury, a reduced heart rate will slow down loss of blood from any 'puncture' wounds. Additionally, not being able 


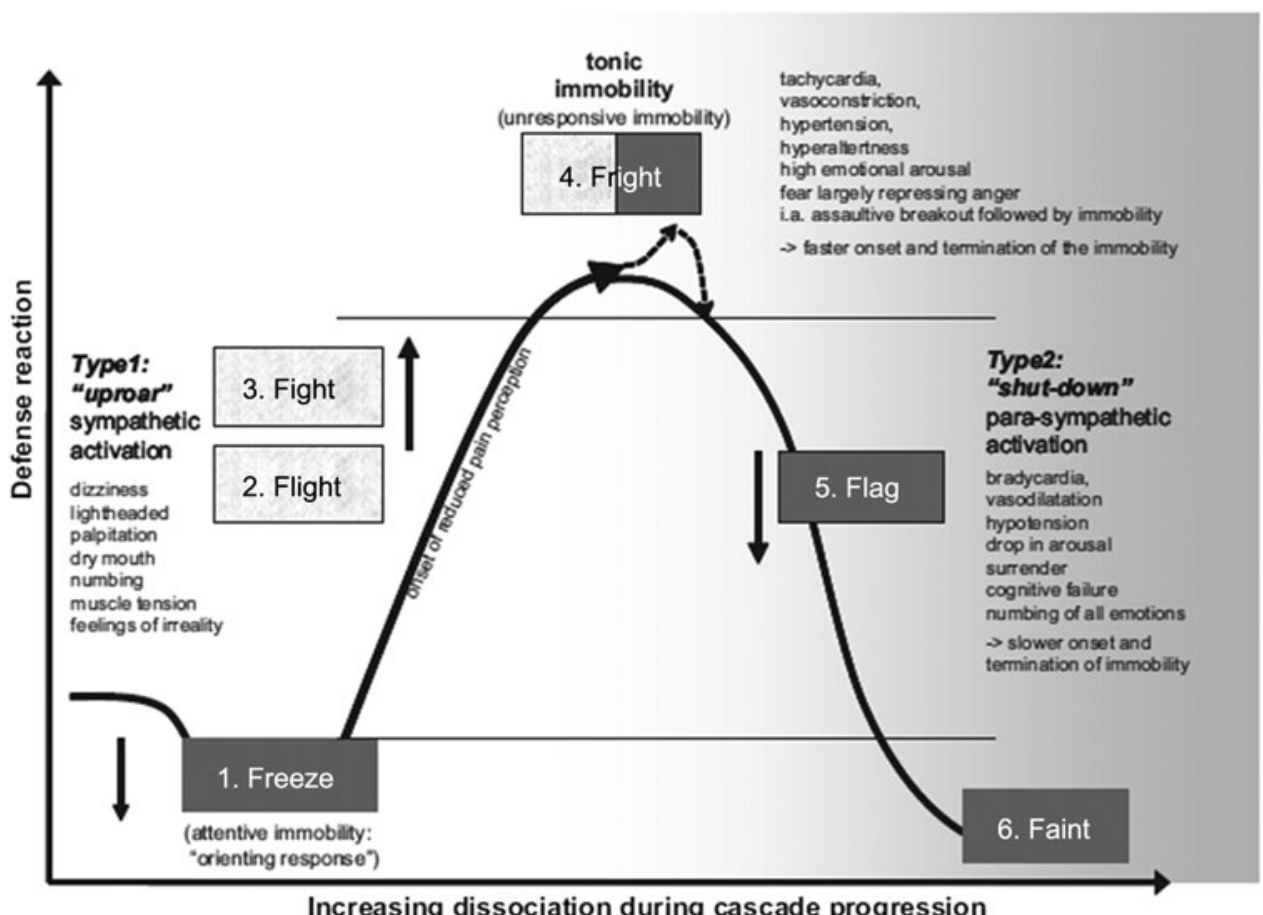

Figure 1. The '6 Fs' cascade. Used with permission from Zeitschrift für Psychologie /Journal of Psychology (2010), vol. 218, pp. 109-127. (C2010 Hogrefe Publishing, www.hogrefe.com; doi: 10.1027/0044-3409/a000018.

to struggle during a sexual assault may minimize further physical injury. Reduced awareness of pain and of any further details of the trauma may also serve to protect the individual from additional emotional distress, should they survive. It is likely that this dissociative process will also disrupt normal memory processing of the event. There is evidence to suggest that dissociative 'shut-down' might become a conditioned response in individuals who experience repeated traumatic events within a similar context (Adenauer et al., 2010; Bolles and Fanselow, 1980). Therefore, individuals who have experienced multiple traumatic events are more likely to rapidly dissociate during any additional traumas that they experience. The model predicts that individuals who experienced the 'shut-down' response during a traumatic event will respond again with dissociative 'shut-down' when later confronted with trauma-related cues, such as matching environmental triggers, or being asked to recall the traumatic event during trauma-focused therapy.

\section{Dissociation protocol}

The proposed protocol (outlined in Box 1) was developed in a specialist National Health Service (NHS) service for forced migrants with PTSD and is based on Schauer and Elbert's (2010) evolutionary explanation of dissociative symptoms in the context of a PTSD diagnosis. The aim of the protocol is to manage dissociative flashbacks, when a client is re-experiencing sensory components of the traumatic event (e.g. smelling blood when there is none present now), as well as managing a dissociative 'shut-down' process. The protocol draws on the ideas regarding the development and maintenance of PTSD and dissociation suggested by Kennerley (1996), Ehlers and Clark (2000), Ehlers et al. (2005), Schauer and Elbert (2010), and Kennedy et al. (2013). Many therapists will be familiar with making cultural adaptations when working with clients who have a history of forced migration. When working cross-culturally, it may be helpful 
Box 1. Dissociation protocol summarizing what the therapist should cover at each stage

\section{Dissociation protocol}

Clients will already have had at least one session focused on psycho-education for PTSD and traumafocused therapy more generally.

\section{Session 1: Psycho-education about dissociation and introduction of grounding materials}

- Re-cap psycho-education of why flashbacks and nightmares occur.

- Explain the '6 Fs' model of dissociation.

- Identify a recent flashback (identify the traumatic event they 'flashed back' to, where they were when the memory was triggered, what were the similarities between the past and present).

- Introduce concept of grounding using focused attention exercise (e.g. noticing all the blue colours in the room).

- Set homework: complete a flashback diary.

\section{Session 2: Introduce a grounding plan}

- Review homework and re-cap previous session.

- Explain the arm wrestle metaphor.

- Introduce grounding materials.

- Go through a three-step plan (identify flashbacks, identify early warning signs, take grounding action).

- Set homework: develop a grounding plan to be used during the week.

\section{Session 3: Practise grounding strategies and troubleshoot. Complete stimulus discrimination if required}

- Review homework and re-cap previous session.

- Review grounding strategies and adapt as necessary.

- Complete stimulus discrimination if required (identify triggers, verbalize differences, draw attention to the differences).

\section{Session 4: Review of progress}

to consider a 'universalist' approach, which focuses on the similarities across cultures, rather than emphasizing the differences (Beck, 2016). Evolutionary understanding of mental health is consistent with this 'universalist' approach, suggesting ways of conceptualizing symptoms that go beyond cultural identity, which are compatible with the core principles of the cognitive behavioural therapy (CBT) approach (Beck, 2016).

The protocol was developed for refugee clients; however, the ideas suggested may be useful for all clients experiencing dissociative flashbacks, whether or not they have a background of forced migration. Furthermore, whilst it focuses on dissociative flashbacks, the strategies may be helpful for clients presenting with other dissociative phenomena, such as derealization and depersonalization, but the protocol does not address these issues directly. The protocol was developed for working with an adult population and the techniques would need adapting if working with children.

The primary aim of this protocol is to reduce the impact of dissociative flashbacks, prior to trauma-focused therapy. Phased-based approaches, encompassing stabilization, trauma-focused therapy and re-integration phases, have become common for the structuring of treatment of refugees in recent years (Herman, 1992; UK Psychological Trauma Society, 2017). Despite stabilization interventions being widely offered, new research and guidance suggests that access to trauma-focused therapy should not be unnecessarily delayed by an extended period of stabilization, unless there is a clear clinical need or risk (De Jongh et al., 2016; UK Psychological Trauma Society, 2017). While some studies have suggested that dissociative symptoms may negatively 
impact outcomes of evidence-based treatments that attempt to integrate the trauma memory into the individual's autobiographical memory (Lanius et al., 2010, 2012; Spitzer et al., 2006), others demonstrate positive outcomes despite dissociation (Hagenaars et al., 2010; Halvorsen et al., 2014; Zoet et al., 2018). It is important therefore to formulate with which clients it would be beneficial to use the dissociation protocol prior to trauma-focused therapy. It is usually clients who would be unable to concentrate and retain information during sessions, or who cannot emotionally engage with their traumatic memories due to the severity of their dissociation, who would benefit most from the protocol. The proposed protocol could be used to help clients manage dissociative symptoms prior to any evidence-based trauma-focused intervention in the second phase [as per the recommendations in the National Institute for Health and Care Excellence (NICE) guidance, 2018], including trauma-focused CBT. Current research suggests that for refugees who have experienced multiple traumas, narrative exposure therapy (NET) (Schauer et al., 2011) has the strongest evidence base (Nosè et al., 2017; Thompson et al., 2018). However, more recently, evidence also indicates that eye movement de-sensitization and re-processing (EMDR) may be an effective treatment for this client group (Thompson et al., 2018).

\section{From theory to practice}

The protocol should be delivered on a one-to-one basis which allows the therapist to individualize the intervention, increasing its effectiveness in helping clients manage their dissociative symptoms. It also avoids the challenges that may come when engaging refugee clients in groups, such as a possible 'language barrier', and difficulties that they may experience interacting with others, due to a lack of trust and/or feelings of shame.

The protocol consists of four sessions, which is based on the average number of sessions found to be clinically effective in the authors' experience, whilst also taking into consideration the pressures on service provision within the NHS. Naturally, some clients will be able to take on board these ideas more quickly, allowing treatment to progress sooner to trauma-focused therapy. Other clients with particularly severe dissociative symptoms may require this protocol to be extended over further sessions.

\section{Assessment of dissociation}

The presence and severity of dissociation should be assessed both through clinical assessment and with the use of relevant measures. Schalinski et al. (2015) have designed a structured interview to assess a person's vulnerability to dissociate as a consequence of exposure to traumatic stressors. This structured interview, the Shutdown Dissociation Scale (Shut-D), is based on the evolutionary model of dissociation used in the proposed protocol (Schauer and Elbert, 2010). This measure therefore maps closely onto the pattern of symptoms suggested by the ' 6 Fs' model of dissociation and focuses primarily on the physiological experience of dissociation. Therapists should also consider assessing dissociation using the Clinician Administered PTSD Scale for DSM-5 (CAPS-5) (Weathers et al., 2013), which includes questions that address depersonalization and derealization, and the Dissociative Experiences Scale (DES) (Carlson and Putnam, 1993).

When working with refugee clients it is important to use caution when administering and interpreting these measures. For example, if a measure has not been officially translated for use in a particular language, it can be expected that the interpretation will not be identical each time, which may influence the client's scores. Moreover, many measures are unlikely to have norms that are standardized for a refugee population and may not be culturally sensitive. Therefore, caution should be used when interpreting a client's score against a clinical cut-off or diagnostic threshold. However, these measures may still provide the therapist and client with useful clinical insight into their symptoms and experiences, and therefore have clinical utility, even if the overall score may 
not be as reliable or valid as when used with other client groups. See the British Psychological Society's (BPS, 2017) guide to working with interpreters for useful guidance on this issue.

\section{Formulation of dissociation}

The starting point in CBT will always be a shared formulation that captures both psychological theory and the individual client's beliefs about their difficulties. This should include the client's personal experiences, as well as any important cultural or spiritual beliefs that may influence their understanding of the problem they are addressing in therapy [see Beck (2016) for further information on cross-cultural formulation]. Perhaps reassuringly for therapists who feel less experienced working with clients from diverse cultural or ethnic backgrounds, there is no requirement for the therapist to be an 'expert' in cross-cultural approaches in order to help clients manage their PTSD or dissociative symptoms effectively. No two clients' formulations will be the same, no matter how many demographic similarities they may share. Remaining curious about the client's own experiences and ideas is essential, enabling enquiry about their background and beliefs.

A helpful approach can be to introduce Schauer and Elbert's (2010) model of dissociation as one possible explanation for the difficulties the client has presented with, and be clear that discussions as to how well this fits with the client's own understanding of their problem are important. This provides the client with the space to bring a different perspective and provides the opportunity to identify and explore issues that the client might see differently.

The client may find it difficult to understand their symptoms and/or feel hopeless about the possibility of gaining control or managing these symptoms more effectively. It is an important first step for the therapist and client to build a shared understanding of the dissociative symptoms and for the therapist to instil hope that things can improve for the client.

\section{Managing dissociation}

\section{Psycho-education}

Prior to beginning the protocol, clients should receive sessions focused on psycho-education regarding PTSD, including normalization of symptoms, discussion of maintenance factors, and a rationale for trauma-focused therapy. It is helpful to remind the client that re-experiencing symptoms, and related dissociation, are occurring because traumatic memories have not been properly 'processed' and that the trauma-focused stage of therapy will aid in processing these memories. At this point it may be necessary for the therapist to differentiate dissociative flashbacks from other phenomena such as intrusive memories, rumination/worry, and other intrusive imagery, to ensure the client has a clear understanding of what the sessions will focus on.

A practical consideration is that if the client is not eating and drinking regularly this is likely to worsen their dissociation. Therefore, before beginning any work managing dissociation it is important to discuss this and ensure that the client has eaten and drunk well prior to the session, and has water available to them during the session.

\section{Explain the ' 6 Fs' model of dissociation}

Therapists should then focus on psycho-education regarding dissociation specifically. The concept of dissociation should be introduced and the client's experience of this explored. It can be helpful to describe this as 'shutting down', 'spacing out', and/or losing a sense of where they are.

The dissociative response should then be explained using the ' 6 Fs' cascade. It is important to normalize this response and emphasize that it was automatic and adaptive at the time of the traumatic event(s) to promote their survival. It should also be explained that because the trauma memories have not been properly 'processed', the dissociative response continues to occur, which is likely to be unhelpful for them currently. The therapist should then discuss each stage of the 
' 6 Fs' cascade with the client and the associated physiological, emotional and cognitive reactions. It is important to highlight that part of the 'Fright' phase of the ' 6 Fs' cascade is 'tonic immobility', which suppresses the ability to move and to be angry, at a point where the individual may have been fully aware of what was occurring. It is possible that the client may have appraisals regarding what they should have done, but did not do, due to being immobile during the traumatic event(s). Normalizing this automatic bodily response, and explaining the reasons why this occurs, may help to challenge cognitive appraisals a client could have made during and after the trauma, such as that they were to blame as they did not fight back.

Following this explanation, it should be collaboratively agreed that the remaining sessions will focus on developing strategies to manage dissociation.

\section{Explain the arm wrestle metaphor}

It is well known that it can be beneficial to use metaphors in CBT to aid clients' understanding (Stott et al., 2010). Whilst the therapist should be mindful of the client's level of education, cultural or religious differences, and examples that may trigger traumatic memories, metaphors can be an extremely useful tool in imparting key therapeutic messages in a memorable, yet simple, way.

Therapists could use an analogy of an arm wrestle to help explain the process that occurs during dissociative flashbacks (see Fig. 2). This involves explaining that when experiencing a flashback it is as if an arm wrestle between the past and the present is occurring in the client's brain. Memories from the past are trying to push their attention back to the past, so what they need to do is help their brain 'win' the arm wrestle by pushing back the other way, making the present 'stronger' than the past. Clients can do this by enhancing sensory stimuli in the present, such as focusing on a strong smell, to remind them where they are, that they are in the present, and that in this moment they are safe.

\section{Grounding strategies}

It is important to then collaboratively develop a grounding plan to manage dissociation.

\section{Step 1 - Gather information}

The first step in managing dissociation is to gather information about a client's flashbacks so that an understanding can be developed about why they are being triggered in a given situation and to what part of the client's trauma history they relate. This can be done by identifying a recent example of a flashback, asking the client to complete a flashback diary over the following week, and/or observing a flashback occurring in session.

Once the client has given an example of a recent flashback, discuss and write down which traumatic event they were 'flashing back' to, the recent situation they were in that triggered this flashback, and all the similarities between the traumatic event and the situation in which the

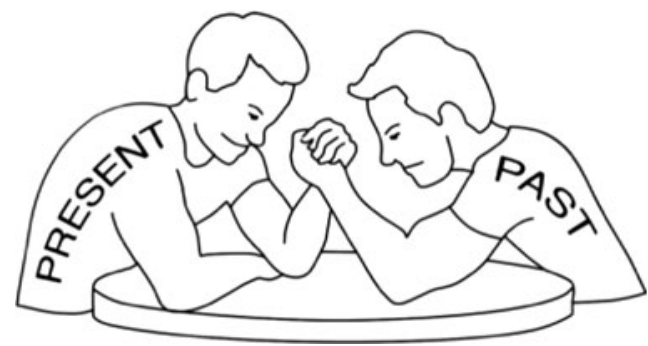

Figure 2. Arm wrestle metaphor diagram. 
Table 1. Example of a completed flashback diary

\begin{tabular}{|c|c|c|}
\hline $\begin{array}{l}\text { What memory is being } \\
\text { triggered? }\end{array}$ & $\begin{array}{l}\text { What were you doing and where were } \\
\text { you? }\end{array}$ & $\begin{array}{l}\text { What is the same as the traumatic } \\
\text { event (triggers)? } \\
\text { (1) People present or how they look } \\
\text { (2) The place } \\
\text { (3) Time of day/year } \\
\text { (4) Objects around you } \\
\text { (5) Sounds } \\
\text { (6) Smells } \\
\text { (8) Taste } \\
\text { (9) What you can see } \\
\text { (10) Feelings/emotions } \\
\text { (11) Physical sensations }\end{array}$ \\
\hline $\begin{array}{l}\text { When they poured petrol } \\
\text { on me }\end{array}$ & $\begin{array}{l}\text { I was walking down the road going to buy } \\
\text { food for my lunch }\end{array}$ & $\begin{array}{l}\text { There were lots of men on the street } \\
\text { I was outside } \\
\text { It was midday } \\
\text { I could feel the hot sun on my skin } \\
\text { I could smell a chemical smell } \\
\text { I could taste a chemical taste in my } \\
\text { mouth } \\
\text { I was thirsty }\end{array}$ \\
\hline
\end{tabular}

flashback occurred. Clients can use flashback diaries between sessions to record flashbacks and the therapist should also model the use of this in session (Table 1).

When this information has been collected it is then easier to identify which stimuli are triggering the client's flashbacks. Stimuli that trigger flashbacks can be specific (e.g. police uniform, sound of sirens, seeing violence on the news) or more indirect (e.g. feeling anxious, feeling angry, changes in body temperature). More indirect triggers can be harder to identify and it can sometimes appear to clients that flashbacks are occurring randomly, which can increase their distress related to PTSD symptoms.

\section{Step 2 - Identify early warning signs}

Once the information about the client's flashbacks has been discussed and triggers identified, the therapist should then explore with the client if they are able to identify any early warning signs that a flashback is about to occur. These signs could be sensations, emotions, and/or visual experiences, often those associated with a drop in blood pressure. If the client finds this difficult, it may help to teach the client mindful attention skills, to help them identify these signs.

However, clients cannot always pinpoint early warning signs and they may feel that there is no warning that a dissociative flashback is about to occur. If this is not easily overcome then do not spend too much time focusing on this, and move on to Step 3. However, if a client is able to identify early warning signs it will allow them to take grounding action as early as possible when they encounter these.

\section{Step 3 - Take grounding action}

Once it has been identified what the client is 'flashing back' to, what triggers this flashback, and if there are any early warning signs, grounding strategies should then be developed. These strategies aim to make the present as attention-grabbing as possible to 'win' the arm wrestle with the past.

Examples of grounding strategies:

- Smells that are pleasant or strong, such as smelling salts, smelling oils, Olbas oil, decongestant sprays/sticks, air fresheners, a favourite perfume, citrus fruits, peppermint, and fresh ginger. 
- Tastes that are strong, such as mints, pastilles, cough sweets, wasabi peas, and chilli.

- Using sight, such as asking the client to notice and describe their environment in detail. For example, the different colours in the room, the lights and shadows, the textures, and any writing they can see. Also consider using clear visual stimuli such as fairy lights. For refugee clients it is useful to use postcards/pictures of scenes associated with the UK (e.g. famous landmarks, the royal family) and Union Jack flags (or items relevant to an alternative host country). The aim of using these stimuli is to remind the client that they are in the present moment, in the relevant year, and in the UK.

- Using touch, such as stress balls, spikey balls, pebbles, fidget cubes, silly putty, marbles, velvet, feathers, natural objects, hot or cold compresses, and Union Jack bracelets.

- Sounds, such as music/sounds that distinguish the present from the past (e.g. comforting relaxation sounds), a loud ticking clock, and radio with English music and voices.

- Using an applied tension technique (Öst et al., 1991) that will increase blood pressure and decrease the likelihood of dissociation. Other activities to increase blood pressure can be beneficial, such as running on the spot, using an exercise bike, standing on a balance board, squeezing stress balls, and/or clenching and unclenching their fists.

- Using postures/actions that are incompatible with the traumatic event, such as a standing posture, walking around, or moving their hands and arms if they had been tied up during the trauma.

- Using cognitive grounding phrases, such as ' $\mathrm{I}$ am in UK, it is Tuesday afternoon, it is 2018 , I can see red buses, I am in the present and not the past, I am safe in this moment'.

One option is to develop grounding strategies for as many senses as possible; however, it is often most effective to match 'sense to sense'. For example, if certain smells regularly trigger flashbacks and/or a client always smells vomit or blood during their flashbacks, it is important to use a strong smell as the initial grounding strategy. The same would be done for a flashback where another sense was primary, such as visual or auditory.

A plan of grounding strategies should also be developed for when a client commonly dissociates when waking following nightmares of the traumatic event(s). These strategies should include placing grounding objects around the room they sleep in, such as postcards/pictures of scenes associated with the UK, Union Jack flags, and importantly lights, enabling them to see the grounding objects when they wake from nightmares. The aim of this is to help the client ground themselves more quickly following nightmares, by reminding them of where there are, and that they are safe. If the client is struggling to implement a grounding plan after waking from a nightmare, the therapist should encourage the client to practise this grounding routine during the daytime, so they are more practised at using this when it is most needed during the night.

It is important to regularly review grounding strategies and change and add to these as necessary. Using grounding strategies between sessions should be discussed, set as a between session task, and a plan made for what the client will use and when. If a client has not managed to do so between appointments, the reasons why this was not completed should be discussed and problem solved. It is likely to be helpful to share this grounding plan with supportive family and friends, to enable them to assist the client in implementing the strategies if they dissociate. The therapist should remind the client that these are strategies to help manage flashbacks and nightmares, but will not prevent them from occurring completely, as it is the trauma-focused therapy that will aid in greatly decreasing the frequency of re-experiencing symptoms.

\section{Stimulus discrimination}

For many clients it may be that the grounding action already discussed is enough to help them better manage dissociative flashbacks and nightmares. However, for some clients with more severe 
dissociation, the stimulus discrimination technique will also be necessary (Ehlers et al., 2005). This technique adds another layer of past/present distinction to the process of grounding.

Following a traumatic event, responses to stimuli that were present during the trauma can generalize, resulting in a client becoming fearful when faced with any environmental stimuli that are similar to those that were present at the time of the traumatic event. For example, if a client was attacked by a tall man on a bus, they may begin to fear tall men on buses, or even all (tall) men. This starts out as an adaptive process called perceptual priming, whereby the traumatized person has a lower threshold for responding to stimuli that were present just before or during the traumatic event. After the event, they are more likely to notice these related stimuli and are more likely to respond with a fear response and accompanying physiological arousal. This is initially an adaptive response, as they attempt to keep themselves safe in the new world they inhabit after they have been involved in trauma. However, if the client's 'alarm system' is constantly triggered when they see a man or travel on a bus, they may end up fearing all men and all public transport. This process of responding with intense fear to more and more triggers that were previously perceived as harmless is called stimulus generalization. This is clearly unhelpful for day-to-day functioning and therefore the stimulus discrimination technique can be used to reduce the number of situations that trigger their alarm response.

The stimulus discrimination technique identifies all the ways in which the stimuli that have triggered the flashback are not the same as the trauma-related stimuli, to reverse stimulus generalization. The aim is to reduce the likelihood of a client having a trauma memory erroneously triggered and/or to stop this process sooner. The steps below are first to be practised in the session, led by the therapist. During sessions, with the therapist's support, it can be helpful to use triggering images or situations to facilitate the client practising these techniques in vivo (see Ehlers et al., 2005). Over time, it is hoped that the client can learn to practise these techniques outside of the session.

\section{Step 1 - Identify triggers}

As when initially developing grounding strategies it is important that the triggers of dissociative flashbacks are identified. This can be done using a stimulus discrimination diary (see Table 2), which is a flashback diary with an additional column used to focus on the differences between the past and present. These diaries can be used to identify which traumatic event the client was 'flashing back' to, the recent situation they were in that triggered this flashback, and all the similarities between the traumatic event and the situation in which the flashback occurred.

\section{Step 2 - Identify differences}

Once a trigger has been identified, the next step is to ask the client to identify all of the differences between the traumatic event and the situation they were in when the flashback was triggered. This can be achieved by asking the client to complete the stimulus discrimination diary.

\section{Step 3 - Draw attention to the differences}

The client's attention should then be drawn to the differences between the traumatic event and the current situation by practising this as a flashback occurs. For example, the client may visit a place that has connections with the trauma, or practise bringing the trauma memory to mind in session. Whilst doing this, the client should verbalize all of the differences. For example, 'this looks different because I can see these men are wearing different clothes, I can see London buses, I am not in my country, and I can move freely'.

It can also be useful to record videos and/or voice recordings of the client and therapist verbalizing these differences, so they can listen to these when in situations in which flashbacks are likely to be triggered. 
Table 2. Example of a completed stimulus discrimination diary

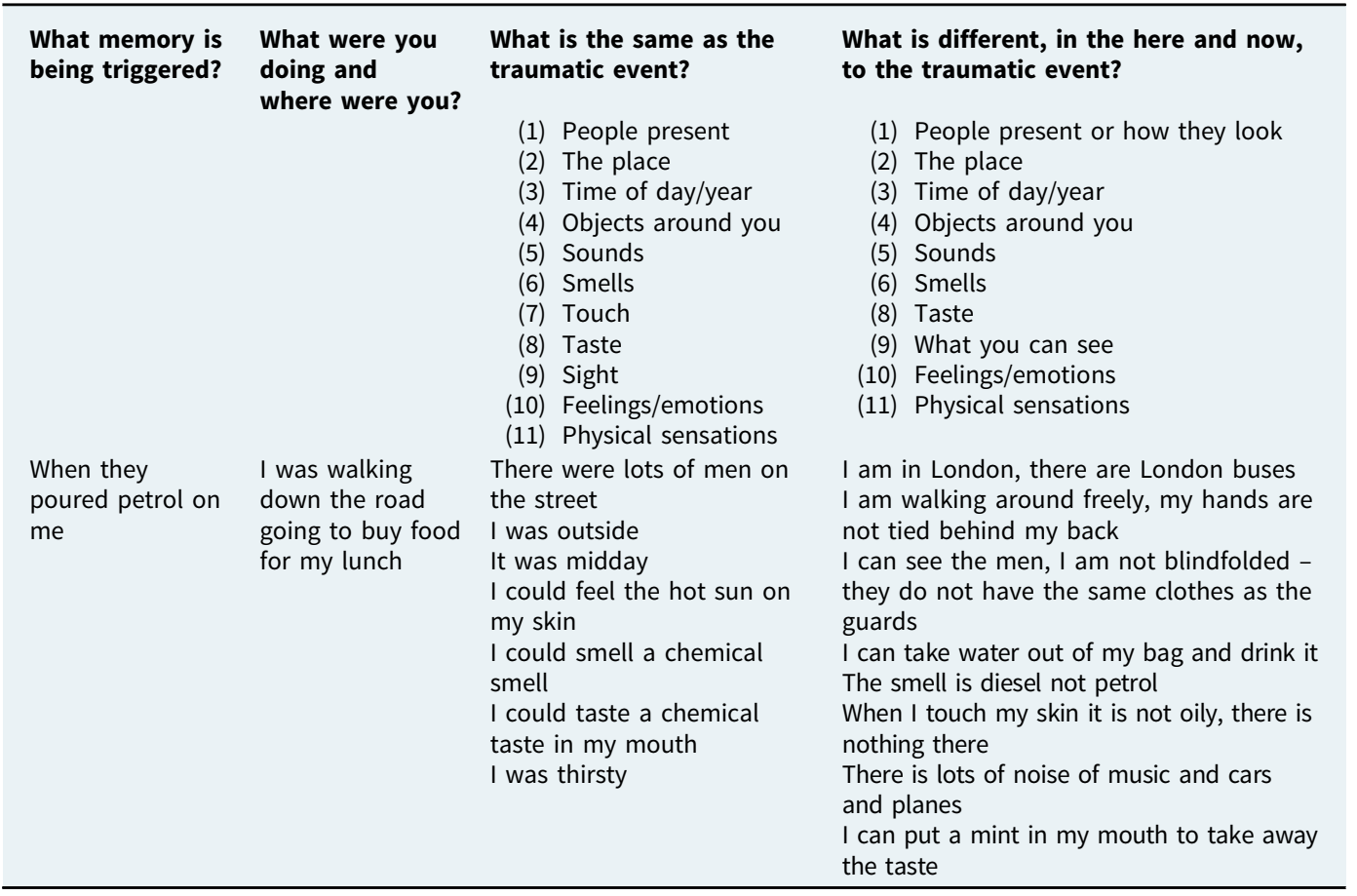

\section{Developing a grounding plan to use during sessions}

In addition to developing grounding strategies to manage dissociation outside of sessions, it is essential to develop a grounding plan to be used in sessions when a client dissociates. This is particularly necessary for trauma-focused therapy, where dissociation is likely to be most frequent. During sessions the therapist should closely monitor the client for any signs of a 'shut-down' dissociative response, such as becoming unresponsive, having an unfocused gaze, a decrease in bodily movements, unclear speech, and/or a delayed or absent response to speech. Usually the client will stop talking and become very still when they dissociate.

Prior to trauma-focused therapy the therapist should discuss with the client how they would like them to respond when they dissociate and what they have found to be most effective previously. It can be beneficial to use grounding strategies that are observable to the therapist, such as the client squeezing a stress ball, clenching and unclenching their fists, or holding one end of a scarf or piece of fabric that the therapist is gently pulling. The idea of this technique is that if the tension in the fabric drops this might indicate that the client is dissociating. These strategies enable the therapist to closely monitor the client's level of dissociation and if necessary encourage them to continue using grounding strategies. For example, if the therapist can see that they have stopped squeezing the stress ball it is likely that their level of dissociation has increased, and they need to be grounded in the room.

While the therapist might be inclined to gently touch the client to ground them quickly when they dissociate in a session, this should be carefully considered, and only used if the client has given prior consent to this. While appropriate touch can be helpful as a grounding technique, if this discussion has not been had prior to doing so, it is possible the use of touch could further trigger traumatic memories of when touch was used in an abusive way. It is essential to agree in advance where on their body you may touch a client during a dissociative episode (e.g. on the 
hand, arm or knee). Alternatively, the therapist can consider saying the client's name, reminding them where they are, and using previously agreed grounding objects, such as smelling oils.

\section{Case study}

Farah is a 35-year-old refugee from Iran referred by her GP. She was imprisoned in Iran for two months and was repeatedly tortured and raped during this time. At assessment, she met diagnostic criteria for PTSD and presented with severe dissociative symptoms. She reported daily dissociative flashbacks to her experiences in prison and described periods of feeling 'absent'.

In the context of a broader formulation of PTSD it was formulated that Farah was experiencing dissociative symptoms because she had regularly dissociated at the time of the traumatic events, and as these memories remained unprocessed, she was experiencing the same 'shut-down' response when reminded (Schauer and Elbert, 2010). It was discussed and agreed with Farah that she would benefit from sessions to help her manage her symptoms of dissociation prior to starting trauma-focused therapy.

In the first session, Farah engaged well with the psycho-education and understood the rationale for learning strategies to manage her dissociation. A flashback diary was used to identify that when she smelt petrol from cars this triggered a specific memory of a traumatic event in prison. A plan was made for Farah to start grounding herself using a strong scented oil. In the second session, Farah reported that she had found this technique useful, but had not always been able to use it in time as it often felt like her flashbacks occurred suddenly. Farah and her therapist spent time identifying an early warning sign of her feeling dizzy and discussed that she would use the grounding objects straight away when she felt this sensation. She also shared her grounding plan with her friends so that they could assist her in using these techniques if they noticed her beginning to dissociate. In the third session, Farah and her therapist discussed stimulus discrimination. They identified another recent situation where Farah had dissociated, when she reported to the Home Office, and explored the differences between her experiences in prison and when she was reporting in the UK. All the differences between these situations were identified and Farah was encouraged to verbalize the differences identified when she next reported, such as people being dressed in different clothes, being able to move her body freely, and that she was in London. By the fourth session, Farah reported feeling more in control when she had a flashback, and was regularly using these techniques both in sessions and at home.

\section{Cross-cultural considerations}

There are a number of additional issues to consider when supporting a refugee client to manage severe dissociative symptoms.

On a practical level, many clients who have a history of forced migration may have unstable legal, social, and financial circumstances. These can have a significant impact on the client's ability to engage with and make use of grounding strategies outside of the therapy session. Many refugee clients will have a severely restricted income and therefore will not be able to afford the practical materials that are suggested. It is beneficial to keep a selection of different types of grounding items that can be given to clients who are not in the financial position to source these themselves. Many refugees will be living in shared, temporary, or transient accommodation. This can make it difficult to set up their environment in order to manage dissociation during the night. In this situation it is necessary to think creatively together about how to set up the client's sleeping environment in order to help them ground themselves at night, despite these barriers. If it is possible to do so, it can help to arrange a visit to the client's home in order to help them set up their grounding materials effectively. 
In addition, the uncertainty and lack of perceived safety in their current circumstances, for example if they have not been granted Refugee Status and so fear deportation to their country of origin, may make it difficult for clients to fully engage with cognitive grounding strategies such as saying to themselves 'I am safe now'. It can be important to be specific with the client, and remind them that whilst they may have other, very valid, anxieties and concerns, the traumatic event causing their dissociative flashbacks is now over. It might be more helpful to use a phrase such as 'I am safe from [the trauma] now. It is in the past'.

The necessity to use an interpreter is a common consideration for therapists working with refugee clients. Reassuringly, evidence suggests that trauma-focused therapy for refugee clients is as effective when delivered via an interpreter as when there is no interpreter present (D'Ardenne et al. 2007). It is best practice to train interpreters in the aims of the protocol and discuss with them how they should helpfully respond during the session if a client dissociates. For example, it should be explained that they continue to interpret what the therapist is saying even if the client does not respond, and they should not try to touch or otherwise comfort the client, and let the therapist take the lead. The therapist should also meet with the interpreter for a short debrief after the session, as is recommended in the BPS guidance for working with interpreters (BPS, 2017).

In addition, this protocol may need to be further adapted for clients who are unable to read or write, including in their mother tongue. Furthermore, clients may also present with (potentially undiagnosed) head injuries or learning difficulties, which may also necessitate adaptions to this protocol. This may include delivering the protocol over a greater number of sessions, more repetitions of the information to facilitate learning, or increasing the use of visual materials to ensure the information is accessible and understood.

Many refugee clients may also be isolated and have limited or no assistance from family or friends to help them implement the strategies at times when they are dissociating. For individuals with very severe dissociation, this can present a challenge. It may require additional work in sessions to help the individual identify early warning signs, select the most effective strategies, and practise using these at times when they are aware of their surroundings until they are over-learnt and can be put into practice even when they are dissociating. If a client's dissociation presents a safeguarding risk to the client or to any children in their care, liaising with other statutory services to ensure they are effectively supported may be necessary.

Overall, whilst cultural and spiritual considerations should be acknowledged and included as part of the grounding strategies where appropriate, it should be emphasized that this type of approach requires no specialist knowledge of the client's culture or background. Curiosity and creativity will be the main tools that will enable therapists to effectively support their clients.

\section{Strengths and limitations}

Strengths of the proposed protocol are that clients have provided feedback that indicates the protocol has good 'face validity' and that the techniques make intuitive sense as a way to address dissociative flashbacks. Qualitative feedback from clients and therapists is often positive about the beneficial impact of these strategies. Furthermore, clients from a range of ethnic and cultural backgrounds, with differing levels of education, are able to engage with the information and the techniques covered during the sessions. Additionally, as the protocol is manualized, more junior members of staff (e.g. Assistant Psychologists) can successfully deliver the protocol with supervision. ${ }^{1}$

A major limitation is that there has been no formal evaluation of the proposed protocol published, although it is currently being evaluated using quantitative rating scales to monitor the outcomes of the intervention. Following the completion of this protocol, clients often report

${ }^{1}$ The full protocol is available upon request by contacting the corresponding author. 
a qualitative sense of improved control over their dissociative symptoms, which facilitates their engagement with trauma-focused therapy. However, this improvement can sometimes be hard to detect using existing standardized measures of dissociation, which may not be sensitive to smaller changes in symptoms. The biggest improvements in clients' dissociative flashbacks are likely to be following trauma-focused therapy. Importantly, the protocol is based on Schauer and Elbert's (2010) model of dissociation, which itself requires further critical appraisal.

Furthermore, the decision regarding a four-session protocol is based on clinical experience and the average number of sessions an individual is likely to benefit from, but is also necessarily limited by pressures on service resources. Therefore, it is possible that the length of this protocol may need to be reviewed in the future.

\section{Summary}

This article outlines a clinical protocol for the assessment, formulation, and management of dissociative symptoms, particularly dissociative flashbacks. It is based on a cross-cultural evolutionary model of dissociation and has been developed with a refugee population, although many of the principles will be applicable for those working with clients with severe dissociative symptoms from any background. This protocol may be helpful in supporting clients with severe dissociative symptoms to subsequently engage effectively in trauma-focused therapy.

Acknowledgments. The authors would like to thank Maggie Schauer for her helpful discussions regarding the ' 6 Fs' model.

Financial support. This research received no specific grant from any funding agency, commercial or not-for-profit sectors.

Conflicts of interest. The authors have no conflicts of interest with respect to this publication.

Ethical statement. The authors have abided by the Ethical Principles of Psychologists and Code of Conduct as set out by the APA. No ethical approval was required for the preparation of this article as it is a practice article and no participants were used.

\section{Further reading}

Beck, A. (2016). Transcultural Cognitive Behaviour Therapy for Anxiety and Depression: A Practical Guide. Oxon, UK: Routledge.

Kennerley, H. (2009). Cognitive therapy for post-traumatic dissociation In A Casebook of Cognitive Therapy for Traumatic Stress Reactions (ed. N. Grey), pp. 93-110. New York, NY, USA: Routledge.

Schauer, M., \& Elbert, T. (2010). Dissociation following traumatic stress: etiology and treatment. Zeitschrift für Psychologie/ Journal of Psychology, 218, 109-127.

\section{References}

Adenauer, H., Catani, C., Keil, J., Aichinger, H., \& Neuner, F. (2010). Is freezing an adaptive reaction to threat? Evidence from heart rate reactivity to emotional pictures in victims of war and torture. Psychophysiology, 47, 315-322.

Ainscough, C., \& Toon, K. (2000). Surviving Childhood Sexual Abuse: Practical Self-Help for Adults that were Sexually Abused as Children. Cambridge, MA, USA: Fisher Books.

American Psychiatric Association (1994). Diagnostic and Statistical Manual of Mental Disorders (4th edn). Washington, DC, USA: American Psychiatric Publishing.

American Psychiatric Association (2013). Diagnostic and Statistical Manual of Mental Disorders (5th edn). London, UK: American Psychiatric Publishing.

Beck, A. (2016). Transcultural Cognitive Behaviour Therapy for Anxiety and Depression: A Practical Guide. Oxon, UK: Routledge.

Bhugra, D., \& Becker, M. (2005). Migration, cultural bereavement and cultural identity. World Psychiatry, 4, 18-24.

Birmes, P., Brunet, A., Carreras, D., Ducasse, J.L., Charlet, J.P., Lauque, D., Sztulman, M.D., \& Schmitt, L. (2003). The predictive power of peritraumatic dissociation and acute stress symptoms for posttraumatic stress symptoms: a threemonth prospective study. American Journal of Psychiatry, 160, 1337-1339. 
Bogic, M., Ajdukovic, D., Bremner, S., Franciskovic, T., Galeazzi, G.M., Kucukalic, A., Lecic-Tosevski, D., Morina, N., Popovski, M., Schützwohl, M., Wang, D., \& Priebe, S. (2012). Factors associated with mental disorders in long-settled war refugees: refugees from the former Yugoslavia in Germany, Italy and the UK. British Journal of Psychiatry, 200, $216-223$.

Bogic, M., Njoku, A., \& Priebe, S. (2015). Long-term mental health of war-refugees: a systematic literature review. BMC International Health and Human Rights, 15, 29-70.

Bolles, R.C., \& Fanselow, M.S. (1980). A perceptual-defense-recuperative model of fear and pain. Behavioural and Brain Sciences, 3, 291-323.

Breh, D.C., \& Seidler, G.H. (2007). Is peritraumatic dissociation a risk factor for PTSD? Journal of Trauma and Dissociation, 8, 53-69.

Brewin, C.R. (2001). A cognitive neuroscience account of posttraumatic stress disorder and its treatment. Behaviour Research and Therapy, 39, 373-393.

British Psychological Society (BPS) (2017). Working with Interpreters: Guidelines for Psychologists. UK: British Psychological Society.

Carlson, E.B., \& Putnam, F.W. (1993). An update on the dissociative experiences scale. Dissociation: Progress in the Dissociative Disorders, 6, 16-27.

Carlson, E.B., \& Rosser-Hogan, R. (1991). Trauma experiences, posttraumatic stress, dissociation, and depression in Cambodian refugees. American Journal of Psychiatry, 148, 1548-1551.

Carlson, E.B., \& Rosser-Hogan, R. (1994). Cross-cultural response to trauma: a study of traumatic experiences and posttraumatic symptoms in Cambodian refugees. Journal of Traumatic Stress, 7, 43-58.

Carswell, K., Blackburn, P., \& Barker, C. (2009). The relationship between trauma, post-migration problems and the psychological well-being of refugees and asylum seekers. International Journal of Social Psychiatry, 57, 107-119.

Chu, J.A. (1998). Rebuilding Shattered Lives: The Responsible Treatment of Complex Post-Traumatic and Dissociative Disorders. New York, USA: Wiley.

D’Ardenne, P., Ruaro, L., Cestari, L., Fakhoury, W., \& Priebe, S. (2007). Does interpreter-mediated CBT with traumatized refugee people work? A comparison of patient outcomes in East London. Behavioural and Cognitive Psychotherapy, 35, 293-301.

De Jongh, A., Resick, P.A., Zoellner, L.A., van Minnen, A., Lee, C.W., Monson, C.M., Foa, E.B., Wheeler, K., ten Broeke, E., Feeney, N., Rauch, S.A.M., Chard, K.M., Mueser, K.T., Sloan, D.M., van der Gaag, M., Rothbaum, B.O., Neuner, F., de Roos, C., Hehenkamp, L.M.J., Rosner, R., \& Bicanic, I.A.E. (2016). Critical analysis of the current treatment guidelines for complex PTSD in adults. Depression and Anxiety, 33, 359-369.

Ehlers, A., \& Clark, D.M. (2000). A cognitive model of posttraumatic stress disorder. Behaviour Research and Therapy, 38, 319-345.

Ehlers, A., Clark, D.M., Hackmann, A., McManus, F., \& Fennell, M. (2005). Cognitive therapy for post-traumatic stress disorder: development and evaluation. Behaviour Research and Therapy, 43, 413-431.

Hagenaars, M.A., van Minnen, A., \& Hoogduin, K.A. (2010). The impact of dissociation and depression on the efficacy of prolonged exposure treatment for PTSD. Behaviour Research and Therapy, 48, 19-27.

Halvorsen, J.Ø., Stenmark, H., Neuner, F., \& Nordahl, H.M. (2014). Does dissociation moderate treatment outcomes of narrative exposure therapy for PTSD? A secondary analysis from a randomized controlled clinical trial. Behaviour Research and Therapy, 57, 21-28.

Herman, J.L. (1992). Trauma and Recovery: The Aftermath of Violence: From Domestic Abuse to Political Terror. UK: Hachette.

Hinton, D.E., \& Lewis-Fernández, R. (2011). The cross-cultural validity of posttraumatic stress disorder: implications for DSM-5. Depression and Anxiety, 28, 783-801.

Kennedy, F., Kennerley, H., \& Pearson, D. (2013). Cognitive Behavioural Approaches to the Understanding and Treatment of Dissociation. New York, NY, USA: Routledge.

Kennerley, H. (1996). Cognitive therapy of dissociative symptoms associated with trauma. British Journal of Clinical Psychology, 35, 325-340.

Kennerley, H. (2009). Cognitive therapy for post-traumatic dissociation. In A Casebook of Cognitive Therapy for Traumatic Stress Reactions (ed. N. Grey), pp. 93-110. New York, NY, USA: Routledge.

Lang, P.J., Davis, M., \& Ohman, A. (2000). Fear and anxiety: animal models and human cognitive psychophysiology. Journal of Affective Disorders, 61, 137-159.

Lanius, R.A., Vermetten, E., Loewenstein, R.J., Brand, B., Schmahl, C., Bremner, J.D., \& Spiegel, D. (2010). Emotion modulation in PTSD: clinical and neurobiological evidence for a dissociative subtype. American Journal of Psychiatry, 167, 640-647.

Lanius, R.A., Brand, B., Vermetten, E., Frewen, P.A., \& Spiegel, D. (2012). The dissociative subtype of posttraumatic stress disorder: rationale, clinical and neurobiological evidence, and implications. Depression and Anxiety, 29, 701-708.

Lewis-Fernandez, R., Martinez-Taboas, A., Sar, V., Patel, S., \& Boatin, A. (2007). The cross-cultural assessment of dissociation. In Cross-Cultural Assessment of Psychological Trauma and PTSD (ed. J.P. Wilson), pp. 279-317. Boston, MA, USA: Springer. 
Li, S.S.Y., Liddell, B.J., \& Nickerson, A. (2016). The relationship between post-migration stress and psychological disorders in refugees and asylum seekers. Current Psychiatry Reports, 18, 82-90.

Miller, K.E., \& Rasmussen, A. (2010). War exposure, daily stressors, and mental health in conflict and post-conflict settings: bridging the divide between trauma-focused and psychosocial frameworks. Social Science \& Medicine, 70, 7-16.

Murray, J., Ehlers, A., \& Mayou, R.A. (2002). Dissociation and post-traumatic stress disorder: two prospective studies of road traffic accident survivors. British Journal of Psychiatry, 180, 363-368.

National Institute for Health and Care Excellence (2018). Post-Traumatic Stress Disorder (NG116). Available at: https:// www.nice.org.uk/guidance/ng116/resources/posttraumatic-stress-disorder-pdf-66141601777861

Nosè, M., Ballette, F., Bighelli, I., Turrini, G., Purgato, M., Tol, W., Priebe, S., \& Barbui, C. (2017). Psychosocial interventions for post-traumatic stress disorder in refugees and asylum seekers resettled in high-income countries: systematic review and meta-analysis. PLoS ONE, 12, e0171030. doi: 10.1371/journal.pone.0171030

Öst, L.G., Fellenius, J., \& Sterner, U. (1991). Applied tension, exposure in vivo, and tension-only in the treatment of blood phobia. Behaviour Research and Therapy, 29, 561-574.

Ozer, E.J., Best, S.R., Lipsey, T.L., \& Weiss, D.S. (2003). Predictors of posttraumatic stress disorder and symptoms in adults: a meta-analysis. Psychological Bulletin, 129, 52-73.

Patel, N., Williams, A.C.D.C., \& Kellezi, B. (2016). Reviewing outcomes of psychological interventions with torture survivors: conceptual, methodological and ethical issues. Torture, 26, 2-16.

Porter, M., \& Haslam, N. (2005). Predisplacement and postdisplacement factors associated with mental health of refugees and internally displaced persons: a meta-analysis. Journal of the American Medical Association, 294, 602-612.

Schauer, M., \& Elbert, T. (2010). Dissociation following traumatic stress: etiology and treatment. Zeitschrift für Psychologie/ Journal of Psychology, 218, 109-127.

Schauer, M., Neuner, F., \& Elbert, T. (2011). Narrative Exposure Therapy: A Short-Term Treatment for Traumatic Stress Disorders (2nd edn). Gottingen, Germany: Hogrefe.

Schalinski, I., Schauer, M., \& Elbert, T. (2015). The shutdown dissociation scale (Shut- D). European Journal of Psychotraumatology, 6, 25652. doi: 10.3402/ejpt.v6.25652

Spitzer, C., Barnow, S., Freyberger, H.J., \& Grabe, H.J. (2006). Recent developments in the theory of dissociation. World Psychiatry, 5, 82-86.

Stott, R., Mansell, W., Salkovskis, P., Lavender, A., \& Cartwright-Hatton, S. (2010). Oxford Guide to Metaphors in CBT: Building Cognitive Bridges. Oxford, UK: Oxford University Press.

Summerfield, D. (2001). Asylum-seekers, refugees and mental health services in the UK. Psychiatric Bulletin, 25, 161-163.

Thompson, C.T., Vidgen, A., \& Roberts, N.P. (2018). Psychological interventions for post-traumatic stress disorder in refugees and asylum seekers: a systematic review and meta-analysis. Clinical Psychology Review, 63, 66-79.

Tinghög, P. (2017). Mental ill-health, trauma and adverse post-migratory experiences among refugees from Syria in Sweden. European Journal of Public Health, 27, 49-50.

UK Psychological Trauma Society (2017). Guideline for the Treatment and Planning of Services for Complex Post-Traumatic Stress Disorder in Adults. UK Psychological Trauma Society.

Weathers, F.W., Blake, D.D., Schnurr, P.P., Kaloupek, D.G., Marx, B.P., \& Keane, T.M. (2013). The ClinicianAdministered PTSD Scale for DSM-5 (CAPS-5). Available at the National Center for PTSD at: www.ptsd.va.gov

Zatzick, D.F., Marmar, C.R., Weiss, D.S., \& Metzler, T. (1994). Does trauma-linked dissociation vary across ethnic groups? Journal of Nervous and Mental Disease, 182, 576-582.

Zoet, H.A., Wagenmans, A., van Minnen, A., \& De Jongh, A (2018). Presence of the dissociative subtype of PTSD does not moderate the outcome of intensive trauma-focused treatment for PTSD. European Journal of Psychotraumatology, 9, 1468707.

Cite this article: Chessell ZJ, Brady F, Akbar S, Stevens A, and Young K. A protocol for managing dissociative symptoms in refugee populations. The Cognitive Behaviour Therapist. https://doi.org/10.1017/S1754470X19000114 